\title{
RELEVANCIA Y SENTIDO DE LA HISTORIA DE LA FILOSOFÍA PARA LA FILOSOFÍA
}

Francisco Abalo

Universidad de Chile

${ }^{1}$ fjabalocea@gmail.com 


\section{PRESENTACIÓN}

El presente volumen es el resultado de un esfuerzo compartido, cuyo origen remonta hacia finales de 2016. La idea de elaborar una publicación que congregara alguna de las muchas voces dentro del mundo académico con el fin de reflexionar sobre el sentido de la historia de la filosofía, fue una idea que surgió en medio de una conversación con Luis Placencia y Miguel Saralegui, al final de un pequeño simposio que dedicamos precisamente a este tema. La realización de este simposio estaba, a su vez, motivada por el afán de darle espacio a una consideración más atenta y reposada a cuestiones que estaban y, de alguna manera, siguen estando presentes en las variadas discusiones acerca de los cambios curriculares dentro de la enseñanza de la filosofía, tanto a nivel secundario como universitario. En el contexto de estas discusiones, para quienes sinceramente están convencidos de la relevancia de la historia de la filosofía, a veces puede resultar ingrato mas no sorprendente, constatar precisamente que esa convicción no es obvia ni necesariamente compartida dentro del mundo de los que están de alguna manera ligados al desarrollo de la filosofía. No es raro que, en el marco cada vez más estrecho de programas disciplinares, se mire con algo de sospecha la presencia de asignaturas que lleven el nombre de "historia de la filosofía", precisamente bajo el supuesto de que no hacen filosofía. Por su parte, es probable que esta sospecha se sustente, en parte, sobre la opinión de que entre la perspectiva filosófica y la histórica se abre una diferencia tajante, y por tanto, la última no pertenece en verdad al conjunto de saberes básicos de la disciplina filosófica.

Es razonable que quienes piensan que la enseñanza de la historia de la filosofía pertenece efectivamente a la formación filosófica no estén dispuestos a poner en cuestión esta convicción en el contexto de discusiones curriculares. Pero tal vez en un marco diferente tiene pleno sentido hacer este cuestionamiento y profundizar así en una dirección que atañe, entre otras cosas, a los supuestos mismos del tipo de ejercicio docente o académico que muchos desarrollamos semestre a semestre durante el curso de nuestras carreras profesionales. De esta forma, la convocatoria a colaborar en lo que llegó a ser este volumen estaba animada por el afán de abrir la pregunta y poder así reflexionar acerca de si tiene alguna relevancia filosófica hacer historia de la filosofía y qué sentido podría tener seguir 
haciéndola. Visto desde este ángulo, la relevancia y el sentido de la historia de la filosofía podría no sólo ser materia de discusión en el contexto de la optimización de la organización de la enseñanza, sino que también una pregunta planteada de tal manera que quienes declaran hacer alguna forma de historia de la filosofía podrían ser llamados a responder no sin cierta filosofía. En definitiva, las preguntas incoadas en el título del presente volumen podrían no sólo tocar a la historia de la filosofía, sino que de modo más o menos directo, apelar también al tipo de concepción filosófica que ve algo importante para su desarrollo en el vínculo con lo transmitido históricamente por su propia disciplina. En las posibles respuestas a la pregunta por el sentido y relevancia de la historia de la filosofía se trata, claro está, de la historia de la filosofía, pero a la vez de la filosofía, o de alguna variedad de ella tal como se ejerce o podría ejercerse hoy.

La pregunta por la relevancia y el sentido de la historia de la filosofía para la filosofía, sugiere una distinción entre historia de la filosofía y filosofía, pero a la vez pregunta por el posible nexo entre ambas. Dispuesta así, la pregunta parece al menos asumir, dicho de una manera un poco tosca, que: 1) es posible hacer filosofía sin remisión a lo transmitido desde alguna de sus múltiples tradiciones, 2) es posible hacer historia de la filosofía sin que su nexo con el ejercicio filosófico tenga peso, 3) es posible hacer historia de la filosofía con filosofía o hacer filosofía haciendo a la vez historia de la filosofía. Las dos primeras posibilidades (hacer filosofía sin historia de la misma; hacer historia de la filosofía sin filosofía), parecen a primera vista fuera de duda. La relación que la filosofía mantendría con su historia sería como la que mantienen otras disciplinas del conocimiento con la posibilidad de hacer historia de esas disciplinas. Así, por ejemplo, es razonable pensar que hacer matemáticas no implica hacer historia de la matemática, y hacer historia de la matemática no es hacer matemática. Incluso más, se puede pensar que hacer matemática implica no hacer historia de la matemática, y hacer historia de la matemática implica no hacer matemática. Lo mismo pasaría con filosofía e historia de la filosofía. Incluso dejando de lado si la comparación con la matemática es la más apropiada, parece ser que este esquema en que el ejercicio de una disciplina excluye la mirada histórica con respecto a esa disciplina, y viceversa, vuelve al menos oscura la tercera posibilidad mencionada antes, o al menos la relega a un territorio cuyos límites son tan difusos que se corre el riesgo de pretender como legítimo un tipo de estudio que a la postre no es sino mala filosofía y mala historia. Ahora bien, tampoco parece tan fácil abandonar esta tercera posibilidad, que es la pretensión que mejor describe lo que muchos declaran hacer, al menos en parte, en el campo de la actividad filosófica actual. De esta manera, es 
pensable que si se presiona reflexivamente en la dirección que marca esta tercera posibilidad, tal vez resulta que la supuesta independencia de las disciplinas implicadas en la expresión "historia de la filosofía" no sea tan obvia como parece en principio, y todo el esquema en que consideramos sus nexos pueda ser repensado desde otras perspectivas.

¿Tiene, entonces, valor filosófico hacer historia de la filosofía para el ejercicio de la filosofía? ¿Bajo qué aspecto podría ser relevante? ¿En qué concepción de la actividad filosófica podría ser valioso el estudio de la tradición? ¿En qué medida nuestra concepción de la filosofía condiciona la selección de los hitos relevantes de la historia de la filosofía (canon)? ¿Hay sólo una forma de hacer filosofía? ¿Hay sólo una tradición filosófica? ¿Hay sólo una forma de hacer historia? ¿Excluye la mirada filosófica la mirada histórica y viceversa, como la tajante distinción entre "historia" y "filosofía" parece asumirlo? ¿Es tan nítida esta distinción cuando la vemos desde la perspectiva concreta de algunas de las múltiples variedades de hacer historia de la filosofía? Estas son algunas de la multitud de preguntas que varios de los trabajos aquí presentados intentan responder desde sus enfoques específicos.

En el primer artículo de este volumen, el profesor Gregorio Piaia, editor y colaborador de uno de los estudios más extensos sobre diversos modelos de historia de la filosofía en el período de la modernidad (Models of History of Philosophy, vol. 2: From Cartesian Age to Brucker), nos recuerda que es de larga data la tensión entre una historia de la filosofía (en sentido subjetivo del genitivo) en que la filosofía provee de los criterios y patrones de juicio para la interpretación de su pasado, una historia especulativa de la filosofía por tanto, y una historia de la filosofía (en sentido objetivo del término) que considera con alta atención los aspectos de contextualización socio-cultural de esta actividad humana (historia histórica de la filosofía). Esta tensión documenta la distinción irreductible entre "hacer filosofía" y "hacer historia de la filosofía". Sin embargo, sostiene Piaia, el intento de resolución de esta tensión, si cabe pretender resolverlo, no tiene por qué llevar a declarar un divorcio entre ambos ejercicios, sino que, si se pone el foco en la concepción filosófica que una historia histórica de la filosofía contiene-filosofía menor acaso, ecléctica y moderadamente escéptica, en la guisa del "comprender" hermenéutico de corte diltheyano-, la convivencia entre ambos enfoques ofrece, entre otras cosas, el potencial de corregir la "tentación del solipsismo y de la autosuficiencia" que asedia al hacer filosofía, cuando se abandona a sí mismo.

Las observaciones que plantea el profesor Piaia permiten considerar con mayor cautela el supuesto de que bajo todo punto de vista en la 
distinción entre "historia filosófica de la filosofía" e "historia histórica de la filosofía", la posición predominante es de la primera, de tal forma que la actividad de la segunda resulte minusvalorada como mera "doxografía". Desde este punto de vista, el profesor Miguel Saralegui, especialista en filosofía política moderna y contemporánea, nos propone mirar con atención esta distinción y los pesos valorativos que parecen estar implicados ahí. El autor toma como marco de análisis el concepto de "investigación", en el sentido de "aumento metódico del conocimiento". Él distingue dos formas de investigación (la intensiva y la extensiva) que corresponden típicamente a ambos modos de hacer historia. Esta perspectiva permite establecer los límites a las pretensiones de la historia filosófica, y realzar las ventajas y aportes de la historia histórica. Propone con ello un modelo de complementariedad en el que sin filosofía no parece poder haber un marco normativo mínimo que oriente la investigación histórica, pero a su vez y tan importante como eso, la historia histórica aporta una mayor amplitud a la autoconcepción que la filosofía detenta en cada momento histórico que desarrolla su actividad.

En estrecho vínculo con algunas precisiones que hace el profesor Saralegui acerca de las diversas formas de relacionarse con el aspecto de institucionalidad que entraña el desarrollo del pensamiento filosófico en su milenaria historia, el profesor Iván de los Ríos presenta una reflexión en torno al carácter y estatuto del canon o del "dinosaurio", para usar su imagen. De los Ríos, quien ha dedicado buena parte de su trabajo a la filosofía antigua, propone considerar la naturaleza del canon filosófico en términos de una "ficción regulativa", pero cuya autoridad no implica una condición metafísica, sino que se configura históricamente a partir del cruce dinámico entre individuos e instituciones, y por tanto como una realidad sujeta a revisión, confrontación y disputa. Lo peculiar en este concepto del canon es que precisamente parece asumir una concepción del quehacer filosófico en que se vuelven esenciales los caracteres de responsabilidad, fragilidad y potencia autocrítica.

Tal vez uno de los supuestos básicos para sostener una neta y hasta excluyente distinción entre filosofía e historia de la filosofía sea la aprensión de perder de vista los problemas a los que hoy debe plantarle cara el ejercicio de la filosofía y, en vez, reemplazar el sentido de esta actividad por un saber de museo. Pero ¿hasta qué punto este juicio no paga el precio de su desconfianza con un grado de generalidad y abstracción que vuelve poco razonable su estimación? En su artículo el profesor Marcelo Boeri, que aparte de su extensa producción en el campo de la filosofía antigua es uno de los autores de la traducción y edición comentada más completa de lo que 
conservamos de la filosofía de los estoicos (al menos, en lengua castellana), ilustra con tres ejemplos la que, a su juicio, es la más convincente de cuatro respuestas posibles a la pregunta “¿por qué trabajar en filosofía antigua?”, a saber, que estudiamos a los antiguos, o al menos a algunos de ellos, porque tienen algo que decirnos acerca de algunos de nuestros problemas filosóficos actuales. El pensamiento de Platón es espacialmente ejemplar para mostrar que el quehacer histórico filosófico no se agota en recoger el aspecto doctrinal de los pensadores del pasado, sino que también ahonda en los temas y problemas que esos filósofos discutieron y trataron de pensar. Boeri expone los rendimientos filosóficos que tiene considerar algunas de las discusiones contenidas en los Diálogos de Platón en relación, por ejemplo, con el problema actual de la teoría tripartita del conocimiento, o con el problema de la autoridad de la primera y la tercera persona, o con el problema del estatuto de la ignorancia en el contexto de la discusión de las virtudes epistémicas. Con ello, se puede sacar la razonable conclusión de que el estudio de la historia de la filosofía contiene, entre otras cosas, un potencial de esclarecimiento y profundización de problemas filosóficos contemporáneos.

Se puede pensar, con ello, que no sólo la distinción entre filosofía e historia de la filosofía resulta muy general, sino que precisamente el estudio de épocas, autores y obras de la tradición filosófica, en cuanto asume y enfatiza la relevancia del vínculo con problemas filosóficos del presente, admite una mayor diferenciación y, por tanto, algún grado de conciencia y reflexión metódica. Este es el punto que desarrolla en su artículo el profesor Luciano Venezia. Aquí se sostiene que se pueden diferenciar tres tipos de ejercicios dentro de lo que él llama el enfoque "analítico" o "sistemático" de las obras clásicas de la filosofía política. Basándose en el tratamiento de cuestiones concretas que el autor ha abordado en varios de sus trabajos dedicados a las obras de Hobbes y Locke (el concepto de autoridad, el problema de la obligación moral y el de la acción voluntaria), Venezia muestra que este tipo de enfoque puede adoptar la reconstrucción, la ilustración y la utilización como tres ejercicios diversos que, por cierto, no se excluyen entre sí. En definitiva, lo que se documenta en cada una de estas formas de abordar la obras clásicas es el esfuerzo por presentar teorías susceptibles de ser desarrolladas y defendidas en el contexto del debate filosófico político actual y, con ello a juicio de autor, el valor de la historia de la filosofía política resulta coincidente con el de la filosofía política misma.

Ahora bien, no necesariamente la diferencia que está a la base de la expresión "historia de la filosofía" tiene que ser, por decirlo así, abono para una querella entre "filósofos" e "historiadores". El profesor Juan Vicente 
Cortés, quien ha consagrado buena parte de su trabajo a la filosofía de Spinoza, considera que el debate al interior de la disciplina de la historia de la filosofía apunta más bien en la dirección de una reflexión metódica que los variados tipos de historiografías filosóficas pueden hacer suya. En el artículo presentado él ensaya este tipo de reflexión con respecto al método arqueológico en la versión de De Libera. El autor aquí defiende la tesis según la cual en al menos esta variante del ejercicio filosófico el interés filosófico está puesto en esclarecer las condiciones histórico conceptuales a partir de la cuales "un pensamiento se vuelve necesario", lo cual implica a su vez una forma específica de cientificidad filosófica.

El profesor Luis Placencia, por su parte, nos propone mirar con más atención el nexo entre filosofía e historia de la filosofía. Frente a algunos modelos de interpretación de la relación entre la filosofía y su historia que no logran dar cuenta plena de la función metódica que tendría la historia de la filosofía para la filosofía, el autor enfoca sus reflexiones en el sentido que podría tener el genitivo en la expresión "historia de la filosofía". De esta manera, Placencia-que ha dedicado varios se su trabajos académica al pensamiento moderno y, de modo destacado, a la filosofía de Kant- destaca el sentido subjetivo en "historia de la filosofía" (la historia que realiza el filósofo), e identifica con ello dos rendimientos metodológicos de este tipo de ejercicio: la posibilidad de contrastar las propias hipótesis en instancias de control robustas como las documenta la tradición del pensamiento, por una parte, y la de autodistanciamiento frente a los propios supuestos y posibilidades de autoengaño, por otra. En ambos casos, la historia de la filosofía entendida desde este ángulo, se convierte en un instrumento de gran potencial para hacerle frente, en parte, al déficit metódico que parece ser inherente a la práctica de la filosofía.

Todos los artículos que presenta este volumen intentan, por ello, responder desde ángulos y orientaciones diferentes algunas de las preguntas que podría despertar el "centauro" de la historia de la filosofía, para tomar en préstamo una expresión con que Burckhart retratara a la composición inversa, i. e, la filosofía de la historia. No parece ser que la variedad de consideraciones posibles sobre esta materia pueda ser reducida a un conjunto de ideas unívocas a las que todo historiador filósofo o filósofo historiador debiese adscribir. Pero, pese a las diferencias muchas veces irreductibles en la concepción del uso filosófico de la historia de la filosofía, parecen haber más aspectos comunes de lo que se manifiesta a primera vista, como creemos que este acotado conjunto de ensayos puede llegar a mostrarlo. 
Para finalizar nos permitimos agradecer a la profesora Gabriela Rossi, anterior directora de la revista, por haber acogido este proyecto con especial entusiasmo, y al equipo editorial por el constante apoyo en el proceso de confección de este material. Vayan también nuestros agradecimientos a todos quienes directa o indirectamente colaboraron para la publicación de esta colección de artículos, en especial al Sr. Carlos Lincopi B., quien se encargó en primera instancia del trabajo de detección de errores y de traspasar los diversos sistemas de citas y formas de presentación de cada colaboración a la norma única con que se presentan aquí. 